\title{
Comparison of P2Y12 receptor inhibition by clopidogrel and prasugrel in patients undergoing percutaneous coronary intervention
}

\author{
Haq $\mathrm{MM}^{1}$, Ahsan $\mathrm{CH}^{2}$, Amin $\mathrm{MN}^{1}$, Karim $\mathrm{MR}^{1}$, Ali $\mathrm{ML}^{1}$, Khan $\mathrm{SR}^{1}$, Chowdhury MZ \\ Mansur $\mathrm{M}^{1}$, Millat $\mathrm{MH}^{1}$, Rashid $\mathrm{MA}^{1}$ \\ ${ }^{1}$ Ibrahim Cardiac Hospital \& Research Institute, Shahbag, Dhaka, Bangladesh, ${ }^{2}$ Department of Cardiology, \\ University Medical Center, Las Vegas, 1800 Charleston Blvd., Nevada, USA. \\ Email: maksumulhaq@gmail,com
}

\begin{abstract}
Dual antiplatelet treatment (DAPT) with aspirin and clopidogrel is vital after percutaneous coronary intervention (PCI). Clopidogrel and prasugrel act on P2Y12 platelet surface receptors. Both these P2Y12 inhibitors are pro-drugs and depend on cytochrome system of the liver for their conversion to active metabolite. There is growing concern regarding suboptimal response in platelet inhibition by clopidogrel. Verify Now system got approval by Federal Drug Administration, USA, for assessing platelet function as its result is almost comparable to gold standard Light Transmission Aggregometry (LTA). There are no data on the prevalence of clopidogrel resistance in Bangladeshi population. Prasugrel, as an antiplatelet drug, is a newer introduction in this country. This study will show light on the efficacy of these drugs on our population especially in patients who undergo PCI where DAPT is mandatory. A total 120 (60 diabetics ) patients with Acute Coronary Syndrome (ACS), were alternatively given $600 \mathrm{mg}$ clopidogrel loading dose (LD) followed by $75 \mathrm{mg}$ maintenance dose (MD) daily or $60 \mathrm{mg}$ LD of prasugrel followed by $10 \mathrm{mg}$ MD daily. Five samples of blood were taken at different time intervals over a period of 2 weeks. Measurement of percent inhibition of P2Y12 was done by VerifyNow. Patients who showed less than $20 \%$ inhibition (clopidogrel resistant) at any stage were switched to prasugrel. The outcomes of clopidogrel, prasugrel and clopidogrel switched to prasugrel groups were then compared. Nearly half $(46.7 \%)$ of the patients in the clopidogrel group was found resistant to the drug as opposed to none in the prasugrel group. No difference was found between diabetic and non-diabetic subjects with respect to drug resistance. Intracoronary blood samples showed high degree of platelet inhibition with prasugrel. There was a gradual decline of platelet inhibition over two weeks with prasugrel. Almost fifty percent of the population is clopidogrel resistant in our study. Prasugrel is a much more potent antiplatelet drug and should be preferred in patients undergoing PCI. Prasugrel may also show resistance over time.
\end{abstract}

\section{Introduction}

Platelet inhibition occurs by a number of mechanisms (Fig A). Aspirin acts intracelluarly by inhibiting cyclooxygenase pathway. Most important platelet surface receptors are P2Y12 and Glycoprotein IIbIIIa (GPIIbIIIa). P2Y12 inhibitors are available as oral form and GPIIbIIIa inhibitors are available as parenteral form.

There are a number of $\mathrm{P} 2 \mathrm{Y} 12$ receptor inhibitors like clopidogrel, prasugrel and ticagrelol. Both clopidogrel and prasugrel are pro drugs, require metabolism by cytochrome system of liver and binds irreversibly to platelet surface P2Y12 receptor. Ticagrelol is not a prodrug and does not require conversion to active form and reversibly binds with the receptor. Dual antiplatelet treatment (DAPT) with aspirin and clopidogrel is currently recommended in patients undergoing $\mathrm{PCI}^{1}$. Despite important clinical benefits of clopidogrel, significant limitations exist ${ }^{2}$. Even with the use of such therapy, a substantial number of subsequent ischemic events may occur ${ }^{\mathbf{3 , 4}}$. Besides, there is interindividual variability in the response to clopidogrel $^{5,6}$. Subjects with suboptimal platelet inhibition by clopidogrel are at increased risk of cardiovascular ischemic events, particularly after $\mathrm{PCI}^{6-9}$. The mechanisms leading to a poor response to clopidogrel have not yet been fully elucidated and are most likely multifactorial. In addition to 
lack of compliance, clinical factors, such as, diabetes, obesity, insulin resistance, food habit concomitant use of other drugs especially proton pump inhibitors and the nature of the coronary events may contribute to the variability of the clopidogrel response $\mathrm{e}^{\mathbf{1 0}}$. There is ample evidence that response to clopidogrel is also influenced by pharmacokinetic variables such as intestinal absorption and metabolic activation in the liver, both of which, in turn, are affected by genetic polymorphisms ${ }^{11-13}$. Patients with type 2 diabetes mellitus (T2DM) are characterized by enhanced platelet reactivity and reduced in vitro responsiveness to antiplatelet agents. Recent studies have shown that T2DM patients have reduced response to $\mathrm{P} 2 \mathrm{Y} 12$ receptor antagonists compared with nondiabetic subjects ${ }^{6,14}$.

Studies of higher loading doses (LDs) and maintenance doses (MDs) of clopidogrel have reported small but statistically significant improvements in the speed of onset, intensity, and consistency of inhibition ${ }^{6,15-17}$. Although there are limited prospective data to support clinical superiority $^{\mathbf{1 8 , 1 9}}$, many clinicians use higher doses of clopidogrel in clinical practice, and recent guidelines support this practice in selected patients $^{\mathbf{2 0 , 2 1}}$. As P2Y12 receptor plays a pivotal role in platelet aggregation, poor platelet response to clopidogrel may be overcome by the use of more potent P2Y12 antagonists or higher doses of clopidogrel $^{22}$.

Many laboratory tests are available to measure platelet function. LTA is still considered the gold standard for assessing platelet function; however it is difficult to set up for common clinical use. The trade names of the tests which are CE marked \& FDA approved are Aggregometry, PFA-100, Verify Now, Plateletworks, Platelet Mapping and Aspirinworks. These tests are performed on whole blood samples and designed for point-of-care testing to provide rapid results ${ }^{23}$. The VerifyNow System (Accumetrics Inc, San Diego, Calif) is a point-of-care turbidimetry-based optical detection system that measures platelet-induced aggregation $^{\mathbf{2 4}}$. The VerifyNow P2Y12 assay has been well correlated with ADP-induced platelet aggregation by $\mathrm{LTA}^{\mathbf{2 5 - 2 7}}$. This system measures platelet-induced aggregation as an increase in light transmittance and uses a proprietary algorithm to report values in P2Y12 reaction units (PRU) and also as percent inhibition. A higher PRU or a lower percent inhibition connotes the same meaning - the lesser inhibition of platelet. PRU value above 208 and percent inhibition less than $20 \%$ is considered as suboptimal response or resistance ${ }^{\mathbf{2 8 - 3 0}}$. The present study was intended to compare the impact of a LD and MD dose of clopidogrel and prasugrel in inducing platelet inhibition in high risk ACS patients undergoing PCI.

\section{Materials and Methods}

The present prospective comparative clinical study was conducted in the Department of Cardiology, Ibrahim Cardiac Hospital \& Research Institute (ICHRI), Dhaka from January 2012. All adult ACS patients ( $\geq 18$ years) with TIMI (Thrombolysis In Myocardial Infarction) score 3 or more and willing to undergo PCI (if needed) were eligible to participate in the study. Patients of ACS with platelet count $<150,000 / \mathrm{cu}-\mathrm{mm}$ or at high risk of bleeding following $\mathrm{LD}$ of clopidogrel or prasugrel or suffering from chronic renal failure (serum creatinine $>2 \mathrm{mg} / \mathrm{dl}$ ) or patients getting prasugrel were excluded from the study.

Blood samples were collected before giving LD of clopidogrel or prasugrel and were kept for maximum of 4 hours (as blood samples kept for more than 4 hours are not recommended for P2Y12 assay) at ICHRI Laboratory. TIMI score was calculated from the following factors, each having score of ' 1 ': a) age $\geq 65$ years, b) $\geq 3$ risk factors for coronary artery disease (CAD) (family history of CAD, hypertension, hypercholesterolemia, diabetes, or current smoker), c) known CAD (stenosis $\geq 50 \%$ ) d) aspirin use in last 7 days, e) severe angina ( $\geq 2$ episodes within last 24 hours) $\mathrm{f}$ ) ST change $(\geq 0.5 \mathrm{~mm})$ in ECG and $\mathrm{g}$ ) positive cardiac marker. Written consent was obtained from each study subjects who voluntarily participated in the study. The study commenced on obtaining approval from the Ethical Review Committee of Ibrahim Cardiac Hospital \& Research Institute.

Based on predefined eligibility criteria, a total 188 patients were initially selected and were given either $600 \mathrm{mg}$ clopidogrel or $60 \mathrm{mg}$ of prasugrel as LD irrespective of their previous intake of clopidogrel. Of the 188 patients 39 underwent coronary artery bypass graft (CABG), 14 refused PCI and 15 withdrew themselves from the study leaving 120 to finally participate in the study. Of the 120 subjects included in the study, 60 were initially assigned to clopidogrel (30 diabetic) and 60 to prasugrel group (30 diabetic). According to study protocol the subjects who exhibited clopidogrel resistance at any stage of the study, were switched to prasugrel, and was treated as another group. Thus, the study subjects finally formed three groups-clopidogrel group $(n=23)$, clopidogrel to prasugrel group $(n=37)$ and prasugrel group $(n=60)$. All the baseline characteristics and 
outcomes were then compared among the three groups. Blood samples from all patients were collected for Troponin-I, blood glucose, HbA1c, S. creatinine, S. electrolytes levels. All patients received aspirin as well. Platelet reactivity was evaluated by the Verify Now P2Y12 assay before LD ( ${ }^{\text {st }}$ sample), 6-24 hours after LD of clopidogrel or prasugrel - just before PCI $\left(2^{\text {nd }}\right.$ sample), during PCI ( $3^{\text {rd }}$ sample from coronary blood after balloon dilatation), 24 hours after PCI ( $4^{\text {th }}$ sample) and 2 weeks after PCI $\left(5^{\text {th }}\right.$ sample). A cut-off value of $<20 \%$ platelet inhibition was considered as resistance. Results were expressed as percent inhibition of P2Y12. Clopidogrel resistant patients were again subdivided into three categories as $0 \%$ inhibition, 1 to $<10 \%$ and 10 to $<20 \%$ inhibition. Statistical analysis was done comparing percent inhibition values among clopidogrel, clopidogrel switched to prasugrel and prasugrel groups.

Data were analyzed using SPSS (SPSS Inc., Chicago, IL, USA), version 16. The test statistics used to analyze the data were Chi-square $\left(\chi^{2}\right)$ or Fisher's Exact Probability Test, McNemer $\chi^{2}$ Test, Student's t-Test and Paired-sample t-Test. The data presented on categorical scale were compared between groups using Chi-square $\left(\chi^{2}\right)$ or Fisher's Exact Test and within group before and after intervention using McNemer $\chi^{2}$ Test, while the data presented on continuous scale were compared among the groups using one-way ANOVA and Repeated Measure ANOVA and were expressed as mean or standard error of mean (SEM). Level of significance was set at 0.05 and $\mathrm{p}<0.05$ was considered significant. The findings obtained from data analysis are documented below.

\section{Results}

Baseline demographic and clinical characteristics of the study subjects are shown in Table I. The subjects in the clopidogrel and clopidogrel switched to prasugrel groups were somewhat older compared to those of prasugrel group. However, prasugrel group had more male (91.6\%) than clopidogrel group (82.6\%) and clopidogrel to prasugrel group $(75.7 \%)$. While the BMI was almost identical among the three groups, diabetic subjects were higher in clopidogrel group $(56.5 \%)$ than those in prasugrel $(50.0 \%)$ and clopidogrel switched to prasugrel group (45.9\%). However, prevalence of hypertension was higher in the latter two groups than the clopidogrel group. The distributions of TIMI score and $\mathrm{HbA1C}$ were almost similar among the three groups. The incidences of unstable angina and LVF were observed to be higher in the switched group compared to clopidogrel and prasugrel group.

Table I: Baseline clinical and laboratory chracteristics

\begin{tabular}{|c|c|c|c|c|}
\hline \multirow{2}{*}{ Variables } & \multicolumn{3}{|c|}{ Group } & \multirow[b]{2}{*}{$\begin{array}{c}\mathrm{p}- \\
\text { value }\end{array}$} \\
\hline & $\begin{array}{c}\text { Clopidogrel } \\
(n=23)\end{array}$ & $\begin{array}{c}\text { Clopidogrel } \\
\text { switched to } \\
\text { Prasugrel }(n=37)\end{array}$ & $\begin{array}{c}\text { Prasugrel } \\
(n=60)\end{array}$ & \\
\hline Age* (years) & $55.09 \pm 11.09$ & $56.92 \pm 11.26$ & $50.78 \pm 8.66$ & 0.312 \\
\hline BMI $^{*}\left(\mathrm{~kg} / \mathrm{m}^{2}\right)$ & $24.30 \pm 4.25$ & $25.10 \pm 2.89$ & $25.91 \pm 3.54$ & 0.830 \\
\hline Male $^{\#}$ & $19(82.6)$ & $28(75.7)$ & 55 (91.6) & 0.095 \\
\hline Diabetes $^{\#}$ & $13(56.5)$ & $17(45.9)$ & $30(50.0)$ & 0.728 \\
\hline Hypertension & $11(47.8)$ & $25(67.6)$ & $40(66.7)$ & 0.228 \\
\hline TIMI score* & $3.61 \pm 0.722$ & $3.97 \pm 0.96$ & 3.920 .99 & 0.794 \\
\hline $\mathrm{HbA} 1 \mathrm{C}^{*}$ & $8.03 \pm 3.17$ & $7.13 \pm 1.34$ & 7.792 .36 & 0.651 \\
\hline \multicolumn{5}{|l|}{ ACS Type $^{\#}$} \\
\hline UA & $10(43.5)$ & $18(48.66)$ & $24(40.0)$ & 0.706 \\
\hline NSTEMI & $13(56.5)$ & $19(51.4)$ & $36(60.0)$ & \\
\hline LVF $^{\#}$ & $1(4.3)$ & $3(8.1)$ & $2(3.3)$ & 0.570 \\
\hline
\end{tabular}

Figures in the parentheses denote corresponding percentage.

* Data were analysed using ANOVA statistics and were presented as mean $\pm \mathrm{SD}$

\#Data were analysed using Chi-square $\left(\chi^{2}\right)$ Test.

Nearly half (46.7\%) of the patients had $<20 \%$ platelet inhibition of platelet measured after 6-24 hours of $600 \mathrm{mg}$ loading dose of clopidogrel. Among the resistant or suboptimal responders, $26.7 \%$ showed $0 \%$ inhibition, $16.7 \%$ showed 1 to $<10 \%$ and $3.3 \% 10$ to $<20 \%$ inhibition (Fig. 1). There was no resistance in the prasugrel group.

Fig 2 compares the percentage inhibition of P2Y12 among the three study groups at five different time intervals. Before loading dose the mean inhibition of P2Y12 in clopidogrel group was $33.17 \%$, in prasugrel group was $19.18 \%$ and clopidogrel switched to prasugrel group was $5.08 \%$. The degree of inhibition 6-24 hours after LD was 53.74\%, $86.8 \%$ and $17.43 \%$, in the three groups respectively. The $3^{\text {rd }}$ sample taken from coronary artery after balloon dilatation before PCI showed reduced percentage inhibition of P2Y12 in all the three groups $(27.26 \%, 60.90 \%$ and $6.14 \%$ in clopidogrel, prasugrel and clopidogrel to prasugrel groups respectively). The percentage inhibition of P2Y12 following PCI (after 24 hours of PCI) was observed to increase again to $56.13 \%, 87.98 \%$ and $55.89 \%$ in clopidogrel, prasugrel and clopidogrel to prasugrel groups respectively, but the increase was significantly steeper in clopidogrel switched to prasugrel group than was observed in clopidogrel and prasugrel groups $(\mathrm{p}<0.001)$. In the next 2 weeks following PCI percentage inhibition of P2Y12 decreased in all the three groups, but it was more so in the prasugrel group than in the other two groups $\quad(p<0.001)$. There were significant differences in P2Y12 inhibition in the three groups in all the five samples of blood collected over a period of two weeks (Table II). 


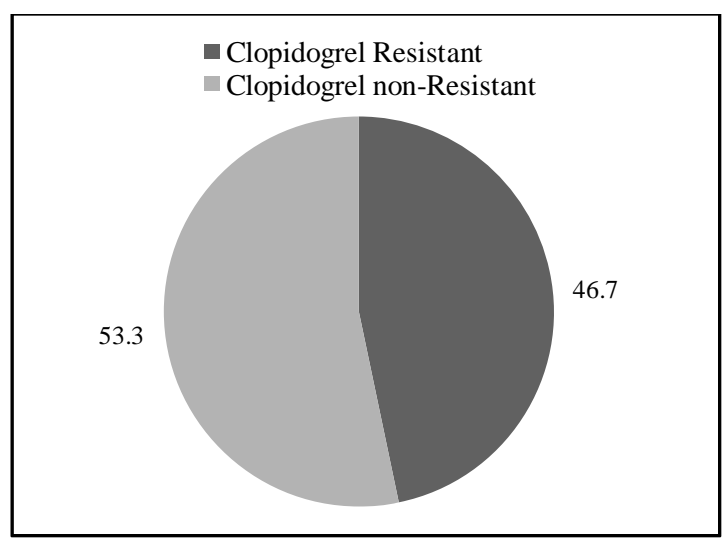

Fig 1: Clopidogrel resistance ( $<20 \%$ inhibition by VerifyNow) measured 2-24 hours after $600 \mathrm{mg}$ of clopidogrel LD

Percentage tratubitcon of P2Y12 in 3 groups at 5 drenent tmes

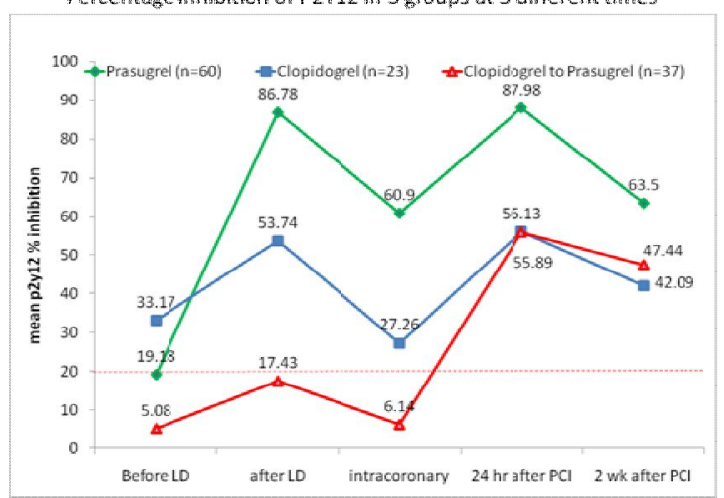

Fig. 2 Platelet Inhibition by clopidogrel, prasugrel and clopidogrel switched to prasugrel before LD, 6-24 $\mathrm{Hr}$ after LD and in intracoronary blood after balloon dilation and 2 weeks after PCI

Table II: Comparison platelet inhibition activities at different time intervals before and intervention anteplatelet drugs

\begin{tabular}{lcccc}
\hline $\begin{array}{l}\text { P2Y12 \% } \\
\text { inhibition* }\end{array}$ & $\begin{array}{c}\text { Clopidogrel } \\
(\mathrm{n}=23)\end{array}$ & $\begin{array}{c}\text { Group } \\
\text { Clopidogrel } \\
\text { switched to } \\
\text { Prasugrel }(\mathrm{n}=37)\end{array}$ & $\begin{array}{c}\text { Prasugrel } \\
(\mathrm{n}=60)\end{array}$ & -value \\
\hline Before LD & $33.17 \pm 3.92$ & $5.08 \pm 1.14$ & $19.18 \pm 2.89$ & $<0.001$ \\
After LD & $53.74 \pm 5.88$ & $17.43 \pm 3.58$ & $86.78 \pm 2.47$ & $<0.001$ \\
Intracoronary & $27.26 \pm 5.53$ & $6.14 \pm 2.58$ & $60.90 \pm 2.81$ & $<0.001$ \\
24-hours & $56.13 \pm 5.58$ & $55.89 \pm 5.53$ & $87.98 \pm 1.47$ & $<0.001$ \\
after PCI & & & & \\
2 weeks after & $42.09 \pm 4.68$ & $47.44 \pm 5.24$ & $63.50 \pm 3.43$ & 0.002 \\
PCI & & & & \\
\hline$*$ Data were analysed using ANOVA statistics and were presented \\
as mean \pm SEM.
\end{tabular}

\section{Discussion}

Risk of all-cause mortality, new or recurrent MI, or severe recurrent ischemia requiring urgent revascularization at 14 days is $13.2 \%$ and $19.9 \%$ if TIMI score is 3 or 4 respectively ${ }^{31}$. The sampled patients who were randomly assigned to clopidogrel and prasugrel had mean TIMI score of more than 3 ,implying that all these patients were at high risk. Clopidogrel resistance has been reported worldwide and varies from country to country and even between study to study within country. The resistance is reported to be high in Asians (>55\%) (because of high genetic polymorphism of the enzyme responsible for conversion of clopidogrel to its active metabolite), compared to that in Whites (30\%) and Blacks $(40 \%)^{32}$.

Lee et al reported that the rate of clopidogrel resistance defined as a \% inhibition $<40 \%$ was $42.9 \%{ }^{33}$. Malinin et al reported that $21 \%$ of the participants had $\%$ inhibition $<30 \%{ }^{24}$. Shim et al reported that the rate of clopidogrel resistance (defined as a percent inhibition $<20 \%$ ) was $40 \%{ }^{28}$ Godino et al reported that the rate of clopidogrel resistance defined as a $\%$ inhibition of $\leq 15 \%$ was $21 \%^{34}$. The wide variation is also partly because of the difference between investigators' options regarding the cut-off value which should be considered as suboptimal and also because of the different time interval taken between drug intake and measurement of blood samples. In our study we took $<20 \%$ inhibition as suboptimal or resistant. The data derived from the analysis showed that nearly half $(46.7 \%)$ of the patients did not achieve $20 \%$ platelet inhibition 6 to 24 hour after $600 \mathrm{mg}$ of LD of clopidogrel. A total of 37 (61.7\%) patients were switched to prasugrel by the investigators at different stages during the two week study period due to suboptimal response. Switching to prasugrel from clopidogrel was done mostly after suboptimal response after LD (46.7\%) and the rest (15\%) after the $4^{\text {th }}$ sample i.e. 24 hours after PCI. If we considered the whole study period of two weeks then clopidogrel resistance is much higher $(61.7 \%)$ compared to any other studies.

Before LD, the mean inhibition of P2Y12 in clopidogrel, prasugrel and clopidogrel switched to prasugrel groups were 33.1, 19.1 and $5.08 \%$ respectively. This was observed because most of the patients were referred to this tertiary care hospital with preloaded clopidogrel. The $3^{\text {rd }}$ sample taken from target coronary artery after balloon inflation showed reduced inhibition in all the three groups which was expected as plaque disruption causes high thrombogenic environment due to activation of platelets.

However, 24 hours after PCI platelet inhibition increased in all 3 groups but the increase was significantly steeper in clopidogrel switched to prasugrel group than was observed in clopidogrel and prasugrel groups $(\mathrm{p}<0.001)$. In the subsequent 2 weeks following PCI percentage inhibition of P2Y12 decreased in all the three groups, but the decrease was significantly faster in the prasugrel group than in the two other groups $(\mathrm{p}<0.001)$. Overall evaluation reveals that prasugrel group exhibited significant improvement from its $19.1 \%$ P2Y12 inhibition at baseline to $63.5 \%$ at end-point of the study, while the clopidogrel group did not 
show any commendable improvement in P2Y12 inhibition activity during the same period (from $33.1 \%$ to $42.1 \%$ ). Clopidogrel switched to prasugrel group, although initially had attenuated P2Y12 inhibition activity, showed dramatic improvement when switched to prasugrel group indicating that clopidogrel-resistant patients are not resistant to prasugrel. However, steady declining trend of P2Y12 inhibition activity in prasugrel group during the 2 weeks period after PCI indicates that, at time, prasugrel group may develop resistance.

Consistent with the findings of the present study, Wiviott and colleagues demonstrated that PCI patients loading with $60 \mathrm{mg}$ prasugrel resulted in greater platelet inhibition than a 600-mg clopidogrel loading dose. Previous reports have shown that suboptimal clopidogrel responsiveness poses considerable risk of ischemic events. ${ }^{35}$ Loading dose $(600 \mathrm{mg})$ have been proposed as a strategy to accelerate and enhance platelet inhibition compared with a standard loading dose of $300 \mathrm{mg}$ and improve short-term clinical outcomes compared with standard clopidogrel therapy. ${ }^{17,36}$ However, findings of the present study show that antiplatelet effects of even $600 \mathrm{mg}$ of clopidogrel loading-dose are not enough in achieving desired therapeutic effect in about half of the patients undergoing PCI where DAPT drugs are essential in preventing stent thrombosis. In addition patients who are suboptimal responders to clopidogrel show high thrombogenic milieu (low p2y12 activity) inside the coronary artery after balloon dilation compared to prasugrel as revealed in the study of the $3^{\text {rd }}$ sample.

As patients rely on their daily maintenance dose of DAPT consisting of aspirin and clopidogrel for prevention of stent thrombosis, present guidelines are emphasizing the role of prasugrel to be superior. Caution should be exercised to prescribe clopidogrel alone in patients who cannot tolerate aspirin as many patients are clopidogrel nonresponders. Our study reveals that improvements in platelet inhibition are transient and confined to the period of high doses of clopidogrel therapy and therefore, long-term maintenance strategy with standard recommended doses seems to be suboptimal in most patients. This is also true for prasugrel, as was evident from the data of percent inhibition of P2Y12 after 24 hours $(87.9 \%)$ and 2 weeks of PCI $(63.5 \%)$.

P2Y12 inhibitor antiplatelet drugs have been used for over 15 years for the prevention of coronary stent thrombosis in patients undergoing percutaneous coronary intervention with stent placement. Now, Prasugrel is available in our country. Ticagrelol and other P2Y12 inhibitor drugs are available in other countries. Although prasugrel is similar to clopidogrel, it is about 10 times more potent and has a quicker onset of action. Data from the largest trial comparing clopidogrel and prasugrel indicate that this increased potency and quicker onset of prasugrel equate to a reduction in major adverse cardiovascular events, although higher rates of major bleeding were reported. Prasugrel also differs from clopidogrel in that it may be less prone to drug-drug interactions and patient nonresponsiveness, although further research is needed in both of these areas. Overall, the data suggest that prasugrel might be a promising treatment option for patients with acute coronary syndromes who are undergoing percutaneous coronary interventions. ${ }^{37}$

Conclusion: From the findings of the study and discussion thereof it can be concluded that in patients of ACS loading dose of prasugrel cause substantial inhibition of platelet function compared to clopidogrel. Clopidogrel resistance was found in nearly fifty percent of the patients. Suboptimal responders after clopidogrel show a staggering improvement in platelet inhibition after switching to prasugrel, indicating that clopidogrel-resistant patients well-responds to prasugrel. Platelet activity inside the coronary artery after balloon dilatation increases due to disruption of plaque causing high thrombogenic milieu where prasugrel is much more superior compared to clopidogrel. The level of inhibition of platelet function by prasugrel gradually decreases over a period of two weeks which raises concern whether long term therapy with prasugrel will lead to a state of suboptimal response. Study on a larger population along with identification of genetic polymorphism of cytochrome enzymes is thought to be necessary to answer these questions.

\section{Acknowledgement}

This study was financed by Bangladesh Medical Research Council (BMRC) during 2012 and ICHRI research fund. We are also thankful to Accumetrics Inc., San Diego, California for providing the Verify Now system and the kits at reduced price for the research purpose.

\section{References}

1. Notarangelo MF, Demola MA, Deigo A. Clopidogrel response variability: clinical applications. Cardiol Clin Prac 2011; 3:91-105.

2. Wiviott SD, Antman EM. Clopidogrel resistance: a new chapter in a fast-moving story. Circulation 2004; 109: 3064-7. 
3. Chen ZM, Jiang LX, Chen YP, et al. Addition of clopidogrel to aspirin in 45,852 patients with acute myocardial infarction: randomised placebo-controlled trial. Lancet 2005; 366:1607-21.

4. Steinhubl SR, Berger PB, Mann JT III, et al. Early and sustained dual oral antiplatelet therapy following percutaneous coronary intervention: a randomized trial. JAMA 2002; 288:2411-20.

5. Angiolillo DJ, Alfonso F. Platelet function testing and cardiovascular outcomes: steps forward in identifying the best predictive measure. Thromb Haemost 2007; 98:707-9.

6. Angiolillo DJ, Fernandez-Ortiz A, Bernardo E, et al. PIA polymorphism and platelet reactivity following clopidogrel loading dose in patients undergoing coronary stent implantation. Blood Coagul Fibrinolysis 2004; 15:89-93.

7. Cuisset T, Frere C, Quilici J, Barbou F, Morange PE, Hovasse T, Bonnet JL, Alessi MC. High post-treatment platelet reactivity identified low-responders to dual antiplatelet therapy at increased risk of recurrent cardiovascular events after stenting for acute coronary syndrome. J Thromb Haemost 2006; 4:542-9.

8. Gurbel PA, Bliden KP, Guyer K, Cho PW, Zaman KA, Kreutz RP, Bassi AK, Tantry US. Platelet reactivity in patients and recurrent events post-stenting: results of the PREPARE POST-STENTING Study. J Am Coll Cardiol 2005; 46:1820-6.

9. Angiolillo DJ, Fernandez-Ortiz A, Bernardo E, et al. Contribution of gene sequence variations of the hepatic cytochrome P450 3A4 enzyme to variability in individual responsiveness to clopidogrel. Arterioscler Thromb Vasc Biol 2006; 26:1895-2000.

10. Karaźniewicz-Łada M, Danielak D, Główka F. Genetic and non-genetic factors affecting the response to clopidogrel therapy. Expert Opin Pharmacother 2012; 13(5): 663-83.

11. Brandt JT, Close SL, Iturria SJ, et al. Common polymorphisms of CYP2C19 and CYP2C9 affect the pharmacokinetic and pharmacodynamic response to clopidogrel but not prasugrel. J Thromb Haemost 2007; 5: 2429-36.

12. Fontana P, Dupont A, Gandrille S, et al. Adenosine diphosphate-induced platelet aggregation is associated with P2Y12 gene sequence variations in healthy subjects. Circulation 2003; 108:989-95.

13. Hulot JS, Bura A, Villard E, et al. Cytochrome P450 2C19 loss-of-function polymorphism is a major determinant of clopidogrel responsiveness in healthy subjects. Blood 2006; 108: 2244-7.

14. Angiolillo DJ, Bernardo E, Ramirez C, Costa MA, Sabate M, Jimenez-Quevedo P, et al. Insulin therapy is associated with platelet dysfunction in patients with type 2 diabetes mellitus on dual oral antiplatelet treatment. J Am Coll Cardiol 2006;48:298-304.

15. Gorchakova O, Schomig A. Loading with $600 \mathrm{mg}$ clopidogrel in patients with coronary artery disease with and without chronic clopidogrel therapy. Circulation 2004; 110:1916-9.
16. Montalescot G, Sideris G, Meuleman C, Bal-dit-Sollier C, Lellouche N, Steg PG, Slama et al. A randomized comparison of high clopidogrel loading doses in patients with non-ST-segment elevation acute coronary syndromes: the ALBION (Assessment of the Best Loading Dose of Clopidogrel to Blunt Platelet Activation, Inflammation and Ongoing Necrosis) trial. J Am Coll Cardiol 2006; 48:931-8.

17. von Beckerath $\mathrm{N}$, Taubert $\mathrm{D}$, Pogatsa-Murray G, Schomig E, Kastrati A, Schomig A. Absorption, metabolization, and antiplatelet effects of 300-, 600-, and 900-mg loading doses of clopidogrel: results of the ISAR-CHOICE (Intracoronary Stenting and Antithrombotic Regimen: Choose Between 3 High Oral Doses for Immediate Clopidogrel Effect) Trial. Circulation 2005; 112:2946-50.

18. Patti G, Colonna G, Pasceri V, Pepe LL, Montinaro A, Di Sciascio G. Randomized trial of high loading dose of clopidogrel for reduction of periprocedural myocardial infarction in patients undergoing coronary intervention: results from the ARMYDA-2 (Antiplatelet Therapy for Reduction of Myocardial Damage During Angioplasty) study. Circulation 2005; 111:2099-106.

19. Wolfram RM, Torguson RL, Hassani SE, Xue Z, Gevorkian N, Pichard AD, Satler LF, Kent KM, Waksman R. Clopidogrel loading dose (300 versus 600 $\mathrm{mg}$ ) strategies for patients with stable angina pectoris subjected to percutaneous coronary intervention. Am J Cardiol 2006; 97:984-9.

20. Silber S, Albertsson P, Aviles FF, Camici PG, Colombo A, Hamm C, Jorgensen E, Marco J, Nordrehaug JE, Ruzyllo W, Urban P, Stone GW, Wijns W. Guidelines for percutaneous coronary interventions: the Task Force for Percutaneous Coronary Interventions of the European Society of Cardiology. Eur Heart J 2005; 26:804-47.

21. Smith SC, Feldman TE, Hirshfeld JW , Jacobs AK, Kern MJ, King SB et al. ACC/AHA/SCAI 2005 guideline update for percutaneous coronary intervention: summary article: a report of the American College of Cardiology/American Heart Association Task Force on Practice Guidelines(ACC/AHA/SCAI Writing Committee to Update the 2001 Guidelines for Percutaneous Coronary Intervention). Catheter Cardiovasc Interv 2006; 67:87-112.

22. Hechler B, Cattaneo M, Gachet C. The P2 receptors in platelet function. Semin Thromb Hemost 2005; 31:150-61.

23. Guyer KE. The present state of aspirin and clopidogrel resistance, Hamostaseologie 2009; 29:285-90.

24. Malinin A, Pokov A, Spergling M, Defranco A, Schwartz K, Schwartz D, Mahmud E, Atar D, Serebruany V. Monitoring platelet inhibition after clopidogrel with the VerifyNow-P2Y12(R) rapid analyzer: the Verify Thrombosis Risk Assessment (VERITAS) study. Thromb Res 2007; 119: 277-84.

25. Paniccia R, Antonucci E, Gori AM, Marcucci R, Giglioli C, Antoniucci D, Gensini GF, Abbate R, Prisco D. Different methodologies for evaluating the 
effect of clopidogrel on platelet function in high-risk coronary artery disease patients. J Thromb Haemost 2007; 5:1839-47.

26. Lordkipanidze M, Pharand C, Nguyen TA, Schampaert E, Diodati JG. Assessment of VerifyNow P2Y12 assay accuracy in evaluating clopidogrel-induced platelet inhibition. Ther Drug Monit 2008; 30:372-8.

27. Jakubowski JA, Payne CD, Li YG, Brandt JT, Small DS, Farid NA, Salazar DE, Winters KJ. The use of the VerifyNow P2Y12 point-of-care device to monitor platelet function across a range of P2Y12 inhibition levels following prasugrel and clopidogrel administration. Thromb Haemost 2008; 99:409-15.

28. Shim CY, Yoon SJ, Park S, et al. The clopidogrel resistance can be attenuated with triple antiplatelet therapy in patients undergoing drug-eluting stents implantation. Int J Cardiol 2009; 134:351-5.

29. Kim JY, Lee K, Shin M, et al. Cilostazol could ameliorate platelet responsiveness to clopidogrel in patients undergoing primary percutaneous coronary intervention. Circ J 2007; 71: 1867-72.

30. Matthew J. Price,Peter B. Berger, Paul S. Teirstein, Jean-François Tanguay etal. Standard- vs High-Dose Clopidogrel Based on Platelet Function Testing After Percutaneous Coronary Intervention The GRAVITAS Randomized Trial. JAMA 2011; 305:1097-1105.

31. Antman EM., Cohen M, Bernink PJ, McCabe CH, Horacek T. The TIMI Risk Score for Unstable Angina/Non-ST Elevation MI: A method for progrnostication and therapeutic decision making. JAMA 2000; 284:835-42.
32. Mega JL, Close SL, Wiviott SD, Shen L, Hockett RD, etal. Cytochrome $\mathrm{P} 450$ genetic polymorphisms and the response to prasugrel: relationship to pharmacokinetic, pharmacodynamic and clinical outcomes. Circulation 2009; 119:2553-60.

33. Lee DH, Arat A, Morsi H, Shaltoni H, Harris JR, Mawad ME. Dual antiplatelet therapy monitoring for neurointerventional procedures using a point-of-care platelet function test: a single-center experience. Am J Neuroradiol 2008; 29:1389-94.

34. Godino C, Mendolicchio L, Figini F, et al. Comparison of VerifyNow-P2Y12 test and Flow Cytometry for monitoring individual platelet response to clopidogrel. What is the cut-off value for identifying patients who are low responders to clopidogrel therapy? Thromb J 2009; 7:4.

35. Gurbel PA, Bliden KP, Hayes KM, Yoho JA, Herzog WR, Tantry US. The relation of dosing to clopidogrel responsiveness and the incidence of high post-treatment platelet aggregation in patients undergoing coronary stenting. J Am Coll Cardiol 2005; 45:1392-6.

36. Hochholzer W, Trenk D, Frundi D, Blanke P, Fischer B, Andris K, Bestehorn HP, Buttner HJ, Neumann FJ. Time dependence of platelet inhibition after a 600-mg loading dose of clopidogrel in a large, unselected cohort of candidates for percutaneous coronary intervention. Circulation 2005; 111: 2560-4.

37. Reinhart KM, White CM, Baker WL. Prasugrel: A Critical Comparison with Clopidogrel. Pharmacotherapy 2009; 29: 1441-51. 